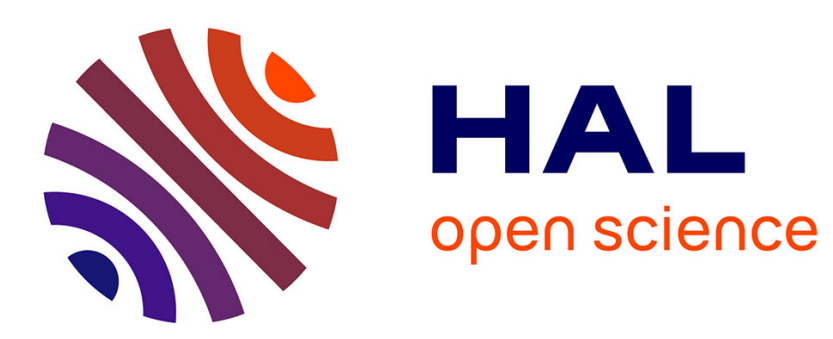

\title{
Données localisées et recherches géographiques en Russie: du problème de la qualité des statistiques russes dans la période de désoviétisation
}

Denis Eckert

\section{- To cite this version:}

Denis Eckert. Données localisées et recherches géographiques en Russie: du problème de la qualité des statistiques russes dans la période de désoviétisation. Espace Géographique, 1993, 10.3406/spgeo.1993.3132 . halshs-02565702

\section{HAL Id: halshs-02565702}

https://shs.hal.science/halshs-02565702

Submitted on 6 May 2020

HAL is a multi-disciplinary open access archive for the deposit and dissemination of scientific research documents, whether they are published or not. The documents may come from teaching and research institutions in France or abroad, or from public or private research centers.
L'archive ouverte pluridisciplinaire HAL, est destinée au dépôt et à la diffusion de documents scientifiques de niveau recherche, publiés ou non, émanant des établissements d'enseignement et de recherche français ou étrangers, des laboratoires publics ou privés. 


\section{Données localisées et recherches géographiques en Russie : du problème de la qualité des statistiques russes dans la période de désoviétisation}

\section{Denis Eckert}

\section{Résumé}

La soudaine abondance de données localisées en provenance de Russie impose de discuter la question de la qualité de l'information statistique russe. Les particularités et les manques de cette information imposent des méthodes de traitement adaptées et des précautions d'emploi, mais ne sont pas une objection à leur utilisation pour la recherche.

\section{Abstract}

Spatially referenced data and geographical research in Russia: the problem of the quality of Russian statistics in a period of desovietisation. - The wealth of spatially referenced data suddenly available from Russia raises questions about the quality of Russian statistical information. The peculia- rites and deficiencies of this information make it necessary to apply appropriate methods and to take certain precautions in processing data but this should not prevent it being used to support research.

\section{Citer ce document / Cite this document :}

Eckert Denis. Données localisées et recherches géographiques en Russie : du problème de la qualité des statistiques russes dans la période de désoviétisation. In: Espace géographique, tome 22, n¹, 1993. pp. 57-62;

doi : https://doi.org/10.3406/spgeo.1993.3132

https://www.persee.fr/doc/spgeo_0046-2497_1993_num_22_1_3132

Fichier pdf généré le 03/01/2019 


\title{
Données localisées ef recherches géographiques en Russie: du problème de la qualité des statistiques russes dans la période de désoviétisation
}

\author{
Denis Eckert
}

CNRS, GIP RECLUS, Montpellier

RÉSUMÉ. - La soudaine abondance de données localisées en provenance de Russie impose de discuter la question de la qualité de l'information statistique russe. Les particularités et les manques de cette information imposent des méthodes de traitement adaptées et des précautions d'emploi, mais ne sont pas une objection à leur utilisation pour la recherche.

INFORMATION STATISTIQUE, QUALITÉ DES DONNÉES, RUSSIE
ABSTRACT. - Spatially referenced data and geographical research in Russia: the problem of the quality of Russian statistics in a period of desovietisation.- The wealth of spatially referenced data suddenly available from Russia raises questions about the quality of Russian statistical information. The peculiarites and deficiencies of this information make it necessary to apply appropriate methods and to take certain precautions in processing data but this should not prevent it being used to support research.

QUALITY OF DATA, RUSSIA, STATISTICAL INFORMATION
La Russie fournit maintenant en abondance données et cartes, et ses chercheurs sont avides de publier dans les revues occidentales. Que penser de cette soudaine production, faut-il s'y fier? Le lourd passé soviétique de manipulation de l'information incite de prime abord à la méfiance. Je crois néanmoins pouvoir fournir des éléments utiles de réflexion, après examen des sources et à l'issue d'un séjour sur le terrain, où j'ai pu travailler sur les données et longuement discuter la question avec des chercheurs russes.
Cet article analyse des problèmes qui se posent pour l'ensemble de la statistique ex-soviétique. Il est néanmoins strictement limité à un examen des perspectives d'évolution des données spécifiquement russes, sans généralisation aux autres États issus de l'ex-URSS. Les évolutions politiques divergentes, les situations de crise aiguë réduisent dès aujourd'hui fortement les possibilités d'analyse globale. La validité des remarques formulées ici ne s'étend pas au-delà des limites de la Fédération russe. 


\section{Ouverture des données ef renouvellement de la recherche}

La période de la «stagnation» brejnévienne avait été marquée par une fermeture quasi paranoïaque du système statistique soviétique. Les données régionalisées n'étaient pratiquement plus accessibles depuis 1975. La perestroika a, là comme dans d'autres domaines, été le signal d'une ouverture remarquée, surtout à partir des années 19881989. Les grands corpus de données régionales ont été ouverts, sur les sujets les plus divers. On a l'impression, après des années de restriction et de sévère contrôle, d'un véritable flot d'information spatialisée.

Cette ouverture, malgré les turbulences politiques et la disparition de l'URSS, ne s'est pas démentie; bon an mal an, les annuaires régionalisés continuent de paraître; les données sont parfois directement disponibles sur disquette. Le Goskomstat prend en compte la question de la diffusion de son information ailleurs qu'en direction des vlasti (Autorités, avec un grand A), ce qui est à tout le moins un changement de comportement.

On prend mieux la mesure de ce changement si l'on se rappelle précisément quelles furent les règles de diffusion et d'exploitation de l'information géographique dans la belle période du «socialisme réel». Après une période de relative ouverture dans les années 1960, on observa une fermeture graduelle, notamment pour les statistiques de production industrielle, qui auraient montré de façon trop manifeste l'échec du rattrapage des États-Unis. Les données spatialisées n'étaient, au moment du brejnévisme, fournies en général que par république de l'exUnion, et au mieux par Grande Région Économique, soit des étendues gigantesques. Elles étaient d'un intérêt fort limité. L'information existait certes à des échelles plus fines, à l'oblast (région) notamment; mais considérée comme confidentielle, elle ne pouvait en général être traitée par un chercheur soviétique qu'à l'occasion de travaux d'expertise effectués pour tel ou tel service de l'État. Il était hors de question d'en tirer la moindre publication, le moindre profit scientifique. Dans certains cas, l'accès à une information jugée sensible (catégorie des plus larges) entraînait même automatiquement une suppression des possibilités de voyage à l'étranger pour cinq ans (1).
Les règles de l'édition scientifique, très contraignantes, imposaient aux auteurs bien davantage que la rituelle préface idéologique et les aussi inoffensives citations régulières, au long de l'ouvrage, des maîtres du marxisme-léninisme. La Geografia promychlennosti SSSR (Géographie industrielle de l'URSS, Khrouchtchev, 1969), ouvrage de la géographie soviétique classique, est emblématique des limitations imposées à l'écriture géographique. Pour présenter les distributions de l'industrie sur le territoire soviétique, l'auteur ne descend jamais au-dessous de la Grande Région Économique; les valeurs correspondant aux classes identifiées dans les légendes des cartes ne sont jamais signalées. On se contente de mentionner que les valeurs sont, pour telle catégorie de régions, «très supérieures à la moyenne», «moyennes», «inférieures», etc. Il était évidemment exclu de tenter une mise en situation internationale sur des variables contrôlables, d'opérer des comparaisons avec d'autres espaces; on aurait nié la spécificité de la «sphère socialiste». Cette mutilation de la pensée n'est d'ailleurs pas à imputer à une autocensure systématique des auteurs. Elle était d'abord le fruit d'une censure "préventive», non officielle, menée de façon active à l'intérieur des maisons d'édition scientifique ellesmêmes. Le «contrôle de conformité» avec les normes idéologiques implicites n'en a pendant longtemps été que plus discret, et plus efficace.

Les géographes russes ont l'immense mérite d'avoir, dans ces conditions, maintenu en vie des écoles vigoureuses, qui ont pu manifester leur qualité dès les débuts de la perestroika.

L'ouverture a permis un remarquable renouveau de la recherche géographique. Ce renouvellement des approches et des thèmes a été évoqué récemment dans L'Espace géographique (Poncé, 1992). La publication de données longtemps retenues dans les bureaux statistiques (données démographiques, par exemple), voire jamais publiées pendant toute la période soviétique (production industrielle des oblasts, grèves, chiffres de la délinquance, des diverses pathologies sociales et médicales...) est une occasion de reprendre des grandes questions à des échelles jusque-là

(1) Entretien avec V. A. Kolossov (Académie des Sciences de Russie), décembre 1992. 
inabordables, de réexaminer les distributions spatiales fondamentales en Russie. L'accès à la base sur les entreprises industrielles de l'ex-URSS (données 1989), concédé il y a deux ans aux géographes de l'Académie des Sciences, fut ainsi un événement. L'exploitation de ces sources libérées a eu pour conséquence une floraison d'articles, limitée malheureusement par la crise de l'édition scientifique en Russie.

En même temps qu'étaient divulguées ces données jusqu'alors retenues sous le vaste coude de la bureaucratie soviétique, une nouvelle information localisée est apparue, liée à la situation politico-économique des dernières années. Une authentique statistique électorale existe maintenant, bien exploitée d'ailleurs, de même que l'on peut obtenir des données sur le processus de privatisation, dans l'agriculture comme dans d'autres secteurs (Néfédova, 1992).

La fin de la pression idéologique a d'autres conséquences. La fièvre comparatiste a saisi les chercheurs russes. Désireux de resituer leur espace dans le contexte européen et mondial, dans son environnement global, ils comparent les données disponibles sur la Russie et ses voisins à celles qui sont produites sur les régions d'Europe occidentale et centrale. Plusieurs articles se font l'écho de cette préoccupation de mise en situation (notamment Gritsaï, 1992). Mais c'est sans doute l'ouvrage Tsentr $i$ Periferia $v$ regionalnom razvitii (Gritsaï, Ioffé, Treivich, 1991) qui est le plus représentatif du bouillonnement comparatiste des dernières années, mouvement impensable sans la libéralisation de l'accès aux donnćcs.

Cette ouverture ne profite pas seulement aux chercheurs issus de l'ex-URSS; les géographes étrangers intéressés ont su participer à l'exploitation de ce gisement d'information, comme l'avait pu C. D. Harris il y a plus de vingt ans (Harris, 1970). L'Atlas de la Population Européenne publié récemment à Bruxelles comprend ainsi de nombreuses cartes incluant les oblasts de la partie européenne de l'exURSS (Decroly, Vanlaer, 1991).

\section{Des données douteuses?}

On comprend l'ardeur des auteurs russes à traiter et mettre en situation leurs données, après tant d'années de sevrage et de censure. Mais avant de se précipiter pour exploiter une information longtemps attendue, de réaliser les traitements statistiques et cartographiques jusque-là impossibles, il convient d'écouter la critique radicale qui met en doute la fiabilité de la statistique soviétique puis post-soviétique. N'est-elle pas sujette à caution, au point d'entacher irrémédiablement d'erreur les analyses qui l'utilisent comme base? Après des années de dissimulation, de tromperie, le système n'est-il pas vicié dans ses fondements et ne se trouve-t-il pas dans l'incapacité, pour longtemps encore, de produire des chiffres fiables? «La transition de l'ancienne Union Soviétique à la nouvelle CEI implique», si l'on écoute les experts occidentaux d'Eurostat, «de tout effacer et de recommencer» (Loening, 1992).

Ce qui vient le plus facilement à l'esprit est la dissimulation de l'importance du complexe militaire dans l'industrie, notamment dans des catégories fourre-tout de l'industrie métallurgique. Mais bien d'autres variables étrangères à la production sont susceptibles d'avoir été manipulées: le soupçon est général.

D'autres défauts, identifiés depuis longtemps déjà, sont liés non à une quelconque tentative de trucage, mais à la structure de l'information elle-même: la statistique des prix à la production, purement arbitraire «ne (fait) généralement qu'enregistrer des prix fixés par l'État...» et fausse toute approche des produits régionaux. La statistique d'entreprise est, selon les critères occidentaux, lourdement défaillante: «leur (comptabilité) s'est limitée presque à de la tenue de stock: combien de voitures produites, combien de pièces fabriquées? La valorisation d'une production ne les intéressait pas. Celle d'une valeur des installations, pas davantage» (ibid.). Et il est vrai que les annuaires de production étourdissent par la précision des listes de quantités d'objets produites par branche et par oblast. La valeur des productions n'était pas une préoccupation des statisticiens, et empêche de se faire une idée de la qualité des productions. On saura que l'oblast de Moscou a produit 197184 microordinateurs au cours de l'année 1990; cette information estelle pour autant la plus pertinente (Goskomstat 1991)?

Parallèlement, le peu d'importance accordé aux services, qui étaient censés n'avoir aucune "production» et n'apparaissaient donc pas dans beaucoup de statistiques, est un sérieux obstacle à une analyse de la tertiarisation de l'appareil économique. 
Et même pour les nouvelles catégories de données, comme les résultats électoraux, peut-on valablement les exploiter, lorsque l'on sait la complexité du jeu politique et les difficultés de formation de l'opinion publique, qui semble interdire toute interprétation des sensibilités politiques des populations? La rapidité du changement n'est-elle pas un défi à l'analyse?

Faut-il à l'extrême, dans ce contexte, s'abstenir d'utiliser les données russes, et attendre la création d'un appareil statistique conforme à nos propres normes, c'est-à-dire peutêtre une dizaine d'années, d'après l'estimation d'Eurostat (Loening, 1992)? Faut-il renoncer à étudier la turbulence de l'espace géographique pendant la transition russe?

\section{Vers une utilisation raisonnée et critique des données}

Les critiques formulées à l'encontre de l'appareil statistique russe, pour justifiées qu'elles soient, n'autorisent sans doute pas pour autant à enterrer toutes les données qu'il produit. C'est peut-être aller un peu vite en besogne, et se priver d'une information précieuse. Les difficultés reconnues de collecte et d'interprétation des données dans le Tiers-Monde n'ont, fort heureusement, pas découragé les géographes de s'y lancer dans des recherches. Qu'il soit permis à la Russie de bénéficier du même droit à l'examen, même s'il doit être accompagné d'un droit (certes aussi limité que possible) à l'erreur.

Si la statistiquc économique a été et reste très éloignée des normes occidentales, la qualité de l'ensemble des données produites par les administrations russes n'est «ni meilleure ni pire qu'ailleurs» (2). La rétention de l'information statistique de nature économique dans l'ex-URSS n'a jamais signifié sa disparition, et ne dit rien sur le travail de collecte lui-même: la culture de la qualité a pu exister chez les statisticiens soviétiques. La pression administrative sur les autorités locales et les entreprises, l'existence de délégués à la statistique dans les unités de production étaient autant d'éléments d'un système de collecte de l'information certes autoritaire, mais assez

(2) Entretien avec A. I. Treivich (Académie des Sciences de Russie), Moscou, novembre 1992 efficace. Ceci est donc plutôt en contradiction avec l'image d'Épinal d'une statistique truquée de la base au sommet. L'intérêt des instituts statistiques et des chercheurs russes est peut-être, aujourd'hui, de convaincre clients ou partenaires étrangers potentiels de la qualité des données qu'ils ont à offrir, de les surestimer pour les rendre plus alléchantes. Il ne faut pas pour autant confondre les données «idéologiques» inventées et diffusées par les bureaucrates des années soviétiques avec celles résultant d'un vrai travail de collecte, dont on dispose aujourd'hui. Ce sont ces dernières sur lesquelles tous les chercheurs, russes ou non russes, auront désormais à travailler. Elles ont certes des défauts structurels, des insuffisances et des trous; mais leur exploitation relève désormais de la classique et rigoureuse critique des sources.

Il est notamment raisonnable de prendre la statistique économique telle qu'elle se présente, dans sa logique et avec ses trous. Cette information a les qualités de ses défauts. La notion de comptabilité régionale n'a guère de sens, ce qui interdit à peu près une approche comparatiste des valeurs de production entre l'Europe occidentale et la Russie. Mais l'importance accordée aux productions et aux flux physiques fait que les données industrielles et agricoles sont souvent originales, et correspondent à des trous de l'information spatialisée en Occident. Des données sur les échanges de produits alimentaires entre les oblasts de Russie existent et promettent des exploitations plus qu'originales sur les liens entre régions. Dans le domaine industriel, on peut espérer suivre de fort près l'effet spatial de la récession qui s'est manifestée à partir de 1991, en suivant les séries régionales de production sur les cinq dernières années (Goskomstat, 1992).

Par ailleurs, les données issues des recensements de population ont toujours été fort convenables. La discussion sur la qualité des données censitaires ex-soviétiques tourne plutôt autour des différences entre millésimes. Là comme ailleurs, certains recensements ont été plus soignés: celui de 1959 a même une excellente réputation, et sert de base pour les analyses en tendance longue (Bond, Treivich, 1993). Certes, les donnécs sur lcs migrations sont généralement considérées comme peu fiables, mais c'est un problème très général que connaissent partout les géographes de la population. 
La meilleure façon de régler la question de la qualité des données est de les recouper. La grande quantité d'information localisée maintenant disponible, collectée par différents services de l'administration russe, autorise en effet de nombreux tests de vraisemblance, et rend bien plus difficiles les tentatives de dissimulation. Il existe à présent une possibilité tout à fait concrète de procéder à un examen critiquc des données, en testant la cohérence interne de l'information disponible, sans attendre qu'une statistique irréprochable tombe du ciel dans un avenir incertain. L'exemple le plus frappant de ces possibilités est fourni par le travail sur le complexe militaro-industriel effectué à l'Institut de géographie de l'Académie des Sciences de Russie. Les géographes de l'IGRAN disposaient d'une base de données sur les «entreprises» industrielles (ce qui correspond plutôt aux unités de production dans le système comptable ex-soviétique), où les entreprises classées dans l'armement n'apparaissaient pas. Cependant, la statistique de l'emploi industriel agrégée à l'unité territoriale (oblast) inclut dans ses totalisations les salariés des entreprises travaillant dans l'armement. Une fois effectuée, pour chaque oblast, la somme $\sum e$ des emplois déclarés par les entreprises (hors secteur militaire), il suffit de faire le rapport $\sum e l e_{0}$ pour chaque oblast (où $e_{0}$ est le nombre d'emplois industriels publié pour chaque oblast) pour obtenir une estimation de la part du secteur militaro-industriel dans chaque région. Pour l'anecdote, le record est détenu par l'Oudmourtie, avec 60\% d'emploi «invisible» (3). On ne peut se bercer d'illusions, et imaginer connaitre par ce moyen tout le complexe militaire. Certains établissements ultrasecrets ne sont encore répertoriés nulle part, et l'imbrication des productions civiles et militaires existe, comme à l'Ouest: une partie de l'effort d'armement est effectuée par des entreprises qui relèvent d'un secteur civil, ce qui fausse les estimations. Mais est-ce une spécificité russe, et la question est-elle parfaitement limpide en Occident? On dit volontiers à Bordeaux que l'activité de la ville est plus grande que la statistique ne le dit, mais qu'on ne peut tout y dire, étant donné la place du complexe militaro-industriel...

La faiblesse de la statistique sur les entreprises tertiaires peut, elle aussi, être en partie compensée. Afin de suivre

(3) Treivich A. I., travail non publié. certaines dynamiques fondamentales de désindustrialisation, notamment dans les espaces métropolitains, on s'attache à retrouver le tertiaire par le biais d'une utilisation des données sur l'emploi; même avec leurs défauts (et notamment une très mauvaise différenciation de l'emploi de production et de l'emploi tertiaire à l'intérieur du secteur industriel), il est possible d'appréhender certaines dynamiques spatiales fondamentales de l'économie (Tarkhov, Treivish, 1992).

Enfin, sur les données directement issues de l'évolution récente (notamment électorales), des formes de traitement ont été imaginées qui prennent en compte l'instabilité et la fragilité des résultats disponibles, et tentent de pallier le manque de recul, l'impression d'indifférenciation et de marais politique, les électeurs semblant se déterminer au hasard. Le Centre de géopolitique européenne de l'Académie des Sciences a pu proposer une typologie électorale des régions de Russie, selon un axe conservatisme-réformisme, en observant les votes des députés du Parlement de Russie. Lors de chaque vote clé permettant aux élus de se différencier politiquement, les comportements des représentants de chaque région ont été notés, ce qui permet ultérieurement d'attribuer une couleur politique à la circonscription qui les a élus. Cette technique, pour audacieuse qu'elle soit, est bien adaptée à des situations de formation de la conscience politique à partir d'un état d'indifférenciation de l'opinion des citoyens. Il s'agit d'un outil d'urgence, appelé à disparaitre dès qu'une série suffisante de votes aura permis de lire directement des résultats électoraux, dès que des candidatures individuelles pourront être clairement attribuées à des partis. Mais il permet d'ores et déjà de formuler de bonnes questions de géographie politique, au lieu d'attendre que cette dérangeante turbulence de la période de transition soit définitivement évacuée.

\section{Incertifudes ef problèmes}

Cependant, de vrais problèmes subsistent, avec des inconnues considérables. Le premier fait acquis est la disparition d'une information cohérente au niveau de l'ex-Union: chaque nouvel État joue sa partition dans son coin. Même à l'échelle de la Russie, la question du maintien d'un appareil statistique efficace est posée, étant donné l'état de désorganisation de l'État central. Déjà, certaines informations 
issues du dernier recensement général de la population soviétique (1989), notamment les séries sur l'emploi, ne seront sans doute jamais publiées.

Fait peut-être moins connu, l'absence de centralisation à Moscou de l'information infra-oblast oblige, pour toute tentative de changement d'échelle, à collccter des données auprès de chaque bureau statistique régional; obtenir dans ces conditions une variable sur un découpage fin pour toute la Russie imposerait donc la visite des quelque 88 capitales d'oblasts et autres républiques autonomes. Ce tourisme statistique est d'ores et déjà pratiqué avec assiduité par les chercheurs russes, mais c'est bien entendu une dépense de temps considérable.

L'évolution politique en cours pose aussi de vrais problèmes: l'autonomisation de fait des régions ne va-t-elle pas affaiblir la production des données localisées? Déjà, la guerre civile rampante dans le Caucase du Nord a empêché le ministère de l'Intérieur de Russie de publier les statistiques de délinquance du Daghestan pour 1992...

Dans un registre moins dramatique, la réorientation et la mise aux normes occidentales de l'appareil statistique va aussi poser des problèmes. La qualité des données sur les productions physiques, certainement l'un des aspects les plus originaux du système soviétique, était assurée par la présence de contrôleurs statistiques dans les entreprises; ils ont aujourd'hui disparu (Loening, 1992). La statistique industrielle va donc passer du régime de l'enregistrement à celui de la réponse à l'enquête. Il n'y a d'ailleurs guère de garantie sur la continuité temporelle des séries: le risque de coupure est très réel pour toutes les données physiques, surtout si les privatisations prennent de l'ampleur. Le type d'information qui pourra être collecté auprès des entreprises privées sera forcément très différent. Avec le secret commercial, la notion de respect du secret statistique au sens occidental (c'est-à-dire la protection des données individualisées) imposera des restrictions. Le suivi méthodique du processus de privatisation par les autorités statistiques apparaît hautement souhaitable: il sera en effet sans doute l'un des meilleurs marqueurs du changement structurel des branches, mais aussi des dynamiques régionales et urbaines (montée des services, par exemple).

\section{Conclusion}

L'information statistique localisée russe ne mérite, à mon sens, ni un excès d'indignité ni un excès de confiance. Il faut la connaître pour mieux savoir la traiter. Les incertitudes sont énormes, mais les enjeux de la recherche sont passionnants. Au prix d'un rigoureux travail sur les données, il est possible d'arriver à des résultats significatifs et valides. C'est peut-être même aujourd'hui surtout qu'il faut travailler, car l'avenir pourrait être plus sombre. Dans ces conditions, pourquoi s'abstenir?

\section{Bibliographie}

BOND A. R., TREIVICH A. I. (1993). "Macro-structural shifts in the former USSR". Post-soviet Geography, à paraître.

DeCroly J. M., VANLAeR J. (1991). Atlas de la Population européenne. Bruxelles: Université de Bruxelles, $172 \mathrm{p}$.

Goskomstat Rossi (1992). Promychlennost' Rossiiskoi Federatsii. Moscou: Respoublikanskii Informatsionno-izdatielskii Tsentr.

GoSKOMSTAT RSFSR (1991). a. Promychlennost' RSFSR v 1990 godu. Moscou: Goskomstat RSFSR; b. Statistika Sotsialnykh Anomalii. Moscou: Goskomstat RSFSR.

GRITSAI O. V. (1992). «Rossia v novom evropeitcheskom mirie: Utopia ili geopolititcheskaiia realnost'». Izvestia Akademii Nauk, seria Geografitcheskaïa, $\mathrm{n}^{\circ} 3$.

Gritsai O. V., Ioffé G.V., TReivich A.T. (1991). Tsentr i Periferiya $v$ regionalnom razvitii. Moscou: Nauka, $168 \mathrm{p}$.

Harris C. D. (1970). Cities of the Soviet Union. Chicago: Association of the American Geographers/Rand Mc Nally, $484 \mathrm{p}$.

KHROUCHTCHEV A. T. (1969). Geografia promychlennosti SSSR. Moscou: Mysl', 438 p.

LOENING K. (1992). «ECO: le grand renouveau». SIGMA, Le bulletin de la statistique européenne, $\mathrm{n}^{\circ} 4$, p. 8-9.

NÉFÉDova T. G. (1992). Sièlskoie Khosiaistvo Rossii nakanunié krupnykh reform 1989-1992. Moscou: IGRAN, 68 p. multigr.

PONCE F. (1992). «Géographes soviétiques: l'après-marxisme». L'Espace géographique, $\mathrm{n}^{\circ} 1, \mathrm{p} .47-50$.

TARKHOV S. A., TREIVISH A. I. (1992). «Geographical location and diffusion of basic innovations: the case of the european USSR». Geo-Journal. 